\title{
Discussing What Would Happen: The Role of Thought Experiments in Galileo's Dialogues
}

\author{
Carla Rita Palmerino*†
}

Thought experiments play an important epistemic, rhetorical, and didactic function in Galileo's dialogues. In some cases, Salviati, Sagredo, and Simplicio agree about what would happen in an imaginary scenario and try to understand whether the predicted outcome is compatible with their respective theoretical assumptions. There are, however, also situations in which the predictions of the three interlocutors turn out to be theory laden. Salviati, Sagredo, and Simplicio not only disagree about what would happen, but they reject one another's solutions as question begging and sometimes even dismiss one another's thought experiments as misleading or nonsensical.

1. Introduction. Although Galileo's name figures virtually in all studies on thought experiments, little attention has been paid to his views on the heuristic and rhetorical power of imaginary scenarios. Kristian Camilleri has recently tried to fill this lacuna by analyzing the role that thought experiments play in Galileo's vision of science. Camilleri offers a classification of Galileo's thought experiments that is based on the different epistemic strategies that he "invoked in trying to address the question of how one can know what would happen in an imaginary scenario" (Camilleri 2015, 103). Despite his oft-repeated warning that "thought experiments are not in themselves sources of knowledge" $(105,111)$, Camilleri seems especially interested in the demonstrative function of Galileo's thought experiments. His main aim is to

\footnotetext{
*To contact the author, please write to: Center for the History of Philosophy and Science, Faculty FTR, Radboud University, PO Box 9103, 6500 HD Nijmegen, The Netherlands; e-mail: c.palmerino@ftr.ru.nl.

$\dagger$ I would like to thank Peter Machamer for organizing the symposium "Galileo and Philosophy of Science"; my fellow speakers Maarten van Dyck, Brian Hepburn, and David Marshall Miller; as well as the participants in the symposium for their questions and comments and the anonymous reviewers for their useful suggestions.
}

Philosophy of Science, 85 (December 2018) pp. 906-918. 0031-8248/2018/8505-0014\$10.00

Copyright 2018 by the Philosophy of Science Association. All rights reserved. 
show how, in the Dialogue, Galileo's spokesman, Salviati, invokes imaginary scenarios in order to "convince" his two interlocutors, the Aristotelian Simplicio and the independently minded Sagredo, "of the truth of a proposition" to which they were "initially unwilling to give assent" (Camilleri 2015, 103).

In this article, I argue that, in Galileo's dialogues, thought experiments function less as demonstrative tools than as magnifying glasses that render the different theoretical assumptions of the three interlocutors visible. Rather than focusing on the conclusions that Galileo draws from his thought experiments, one should, I suggest, look at the discussions that Sagredo, Salviati, and Simplicio conduct and at the arguments they use to motivate their predictions about what would happen in a given imaginary situation.

I first discuss some examples in which the three characters agree about what would happen in an imaginary scenario and try to understand whether the predicted outcome is consistent with their respective theoretical assumptions. Then I present a few cases in which their predictions differ. As we will see, in these cases Salviati, Sagredo, and Simplicio accuse one another of begging the question in the evaluation of a thought experiment and sometimes even refuse to make a prediction on a scenario described by the interlocutor.

2. Agreeing on What Would Happen. Galileo would have certainly agreed with Murdoch (1982) and Grant (2002) that scholastic natural philosophy had lost touch with nature, espousing a type of "empiricism without observation." In his writings, Galileo routinely accuses his scholastic opponents of basing their conclusions on Aristotle's ideas rather than on a careful observation of the book of nature.

This is what happens, for example, in a passage of the second day of the Dialogue, where Salviati criticizes Simplicio for endorsing Aristotle's view that the motion of projectiles is due to the propelling action of the medium. How can Simplicio be "so credulous" when he has "his own senses to refute this claim?" Does he not see that if projectile motion were really caused by the medium, as he believes, then light bodies should move faster and farther than heavy ones? Simplicio has not yet answered these questions, when Sagredo intervenes in the discussion to present an imaginary scenario.

SAGREDO: There is another point of Aristotle's which I should like to understand, and I beg Simplicio to oblige me with an answer. If two arrows were shot with the same bow, one in the usual way and one sideways - that is, putting the arrow lengthwise along the cord and shooting it that way - I should like to know which one would go the farther? Please reply, even though the question may seem to you more ridiculous than otherwise. (Galilei 1967, 152) 
Simplicio replies that although he has "never seen an arrow shot sideways," he thinks "it would not go even one-twentieth the distance of one shot point first." Sagredo agrees with this conclusion but emphasizes that it contradicts Aristotle's account of projectile motion:

SAGREDO: Since this is just what I thought, it gives me occasion to raise a question between Aristotle's dictum and experience. For as to experience, if I were to place arrows upon that table when a strong wind was blowing, one in the direction of the wind and the other across it, the wind would quickly carry away the latter and leave the former. Now apparently the same ought to happen with two shots from a bow, if Aristotle's doctrine were true, because the one going sideways would be spurred on by a great quantity of air moved by the bowstring - as much as the whole length of the arrow-whereas the other arrow would receive the impulse from only as much air as there is in the tiny circle of its thickness. I cannot imagine the cause of such a disparity, and I should like very much to know it. (Galilei 1967, 153)

Here, as in many other passages of the Dialogue, Galileo suggests that the only way to reason about situations that one cannot directly observe, is to explain them by analogy with more familiar phenomena (Palmerino 2011, 108). If air were responsible for the motion of projectiles, then the two arrows shot with a bow should display the same behavior as the two arrows carried away by the wind. Simplicio rebuts that there is an "obvious" reason why this is not the case: "it is because the arrow shot point foremost has to penetrate only a small quantity of air, and the other has to cleave as much as its whole length" (Galilei 1967, 153). As Sagredo points out, however, this answer is at odds with Simplicio's proposed explanation of projectile motion:

SAGREDO: Oh, so when arrows are shot they have to penetrate the air? If the air goes with them, or rather if it is the very thing which conducts them, what penetration can there be? Do you not see that in such a manner the arrow would be moving faster than the air? Now what conferred this greater velocity upon the arrow? Do you mean it to say that air gives it a greater speed than its own? (Galilei 1967, 153)

What Sagredo has in mind is obviously Aristotle's notion of antiperistasis, or "circulating thrust" (Physics 215a15), according to which a projectile divides the air, which then rushes from the front to the back of the projectile pushing it forward. Simplicio's claim that the arrow has to penetrate the air suggests that the air is slower than the arrow and can therefore not be the cause of its motion. Sagredo hence concludes "that the whole thing takes 
place just exactly the opposite to what Aristotle says, and that is as false that the medium confers motion upon the projectile as it is true that it is this alone which impedes it" (Galilei 1967, 153). Simplicio's guess that the arrow shot sideways would be slower than the arrow shot point first is in agreement with everyday experience, which shows that the medium offers resistance to motion, but not with Aristotle's theory of projectile motion, which regards the medium as a propelling force. This gives to Salviati the occasion to point out that in Aristotle's natural philosophy, there are "many propositions . . . that are not only wrong, but wrong in such a way that their diametrical opposites are true" (Galilei 1967, 153).

In the Dialogue, Aristotle's theory of free fall is presented as equally implausible and counterintuitive as his explanation of projectile motion. When, in a passage of the second day, Simplicio endorses Aristotle's claim that "the speeds of falling heavy bodies have among themselves the same proportions as their weights," Salviati invites him to imagine "a hundred-pound ball and a one-pound ball of the same material being dropped at the same moment from a height of one hundred yards." Salviati does not believe that Simplicio can "picture in his mind the large ball striking the ground while the small one is less than a yard from the top of the tower" (Galilei 1967, 223). The Aristotelian does not get the chance to reply, as Sagredo intervenes in the discussion steering it in a new direction.

A reply to Salviati's argument was offered by an Aristotelian in flesh and blood. Antonio Rocco, in his Esercitazioni filosofiche (1633) written in reaction to the Dialogue, endorsed Aristotle's view and challenged Galileo to demonstrate not with experience but with reason that the speed of fall cannot be proportional to the weight of the falling body (Galilei 1890-1909, 7:687). Galileo penned some Postille to the Esercitazioni, in which he described "the intellectual repugnance" he had experienced upon first reading Aristotle's treatment of the problem (Physics 215a25-28) and recalled having formulated a counterargument. He imagined two bricks of equal size and weight that are dropped together from a high tower and, during their fall, all of a sudden join together. Galileo argued that it would be absurd to suppose that the speed of the joined bricks suddenly doubled, given that neither of the two was able to add an impetus to the other. Galileo then proceeded to a "more stringent demonstration" that consisted in imagining the joint fall of two bodies A and B, the first of which is "naturally faster" than the second one. "The union of A and B will result in a compound of speeds greater than the speed of B alone, but smaller than the speed of $\mathrm{A}$ alone; but given that the compound of $\mathrm{A}$ and $\mathrm{B}$ is greater than $\mathrm{A}$ alone, it follows that the greater mobile $\mathrm{AB}$ will move less fast than the smaller mobile B; which is against the supposition" (Galilei 1890-1909, 7:732; my translation).

Aristotle's theory of the proportionality between speed and weight is discussed once again in the Two New Sciences, where Galileo reworks the argu- 
ment of the Postille into what has become one of the most famous thought experiments in the history of science.

In the first day of that work, Salviati explicitly questions that "Aristotle ever tested whether it is true that two stones, one ten times as heavy as the other, both released at the same instant to fall from a height, say, of one hundred braccia, differed so much in their speeds that upon the arrival of the larger stone upon the ground, the other would be found to have descended no more than ten braccia" (Galilei 1989, 66). Simplicio reacts by pointing out that Aristotle's language in Physics 215a25-28_ “we see that the velocity of a moving weight or mass depends on . . . the comparative gravity or levity of the moving body itself" (Aristotle 1929, 351)—seems to indicate that he made the experiment. As was to be expected, the interlocutors are not satisfied with this answer. Sagredo invokes the result of a test he himself has made, whereas Salviati prefers to appeal to the following imaginary scenario:

SALVIATI: But [even] without other experiences, by a short and conclusive demonstration, we can prove clearly that it is not true that a heavier body is moved more swiftly than another, less heavy, these being of the same material. .. . Tell me, Simplicio, whether you assume that for every heavy falling body there is a speed determined by nature such that this cannot be increased or diminished except by using force or opposing some impediment to it.

SIMPLICIO: There can be no doubt that a given body in a given medium has an established speed determined by nature, which cannot be increased except by conferring on it some new impetus, nor diminished save by some impediment that retards it.

SALVIATI: Then if we had two bodies whose natural speeds were unequal, it is evident that were we to connect the slower to the faster, the latter would be partly retarded by the slower, and this would be partly speeded up by the faster. Do you not agree with me in this opinion? SIMPLICIO: It seems to me that this would undoubtedly follow. (Galilei 1989, 66-67)

Salviati's next argumentative step consists in pointing out that Simplicio's prediction conflicts with Aristotle's hypothesis that the speed of fall is proportional to the weight:

SAlviati: But if this is true, and if a large stone moves with a speed of, say, eight while a smaller moves with a speed of four, then when they are united, the system will move with a speed less than eight; but the two stones when tied together make a stone larger than that which before moved with a speed of eight. Hence the heavier body moves with less speed than the lighter; an effect which is contrary to your supposition. 
Thus you see how, from your assumption that the heavier body moves more rapidly than the lighter one, I infer that the heavier body moves more slowly. (Galilei 1989, 67)

Salviati makes use here of an argument ex concessione. He grants to Simplicio an assumption to which he himself does not subscribe, that is, that the speed of a falling body is proportional to its weight, and draws from it a conclusion "unwelcome to the opponent" (Jardine 1991, 113).

There has been much discussion in the literature about the cogency of Galileo's argument. Brown $(2000,528)$ has singled it out as a perfect example of a Platonic thought experiment, which "gives a grip on nature just by thinking." Norton $(1996,2004)$ has used it to prove, against Brown, that thought experiments are just logical arguments in disguise. Gendler (1998) has used it to argue, against Norton, that thought experiments can lead to rationally justified conclusions that would not be rationally justifiable on the basis of a straightforward argument. Whereas Atkinson and Peijnenburg (2004, 127) have maintained, against Gendler, that Galileo's argument is "logically deficient" and fails "to adequately describe the empirical world."

Palmieri has rightly pointed out that scholars interested in "the epistemic status" of past thought experiments should be careful not to examine them according to "a modern frame of mind." What "might today be seen as an instance of a very special form of cognition . . . was born in the murky waters of analogical thinking, ex post facto cognitive autobiography, and, in all likelihood, real experimentation" (Palmieri 2005, 238). The "cognitive autobiography" mentioned by Palmieri is the Postille to Antonio Rocco, in which, as we have seen, Galileo "reconstructs" an old argument of his against Aristotle's theory.

I have little to add to Palmieri's reconstruction of the historical and theoretical context in which Galileo's thought experiment originated. For the current purposes, it suffices to notice that when recasting the reductio ad absurdum argument of the Postille in the Two New Sciences, Galileo scripts a Socratic dialogue in which Simplicio endorses Salviati's conclusion that the faster body would be partly retarded by the slower and the slower partly sped up by the faster. We have here a good example of what Cantor $(1989,172)$ has called a "literary road to consensus." The fact that Simplicio provides no motivation for his belief adds persuasiveness to the counterargument, making the conclusion appear obvious to the reader. At this point, it is easy for Salviati to show that Simplicio's prediction is "contrary to his supposition." For if bodies fell with a speed that was proportional to their weight, then the two bodies tied together should fall faster than either of the two on its own.

Galileo uses a similar argumentative strategy in his criticism of Aristotle's causal explanation of the motion of fall. In the second day of the Dialogue, Simplicio argues, following Aristotle, that heavy bodies are drawn 
by an internal principle toward "the centre of the universe," which by accident coincides with the center of the earth. The motion of fall is therefore natural, whereas the motion upward is per definition preternatural and can only be caused by an external principle. In order to convince his interlocutor that the "principle which moves heavy projectiles upward ... is no less internal and natural than that which moves them downward" (Galilei 1967, 345), Salviati asks him to imagine that "the terrestrial globe were pierced by a hole which passed through its centre" and a cannon ball were dropped into this hole.

Salviati: Having arrived at the centre is it your belief that it would pass on beyond, or that it would immediately stop its motion there? Simplicio: I think it would keep on going a long way.

Salviati: Now wouldn't this motion beyond the centre be upward, and according to what you have said preternatural and constrained? But upon what other principle will you make it depend, other than that which you have already called intrinsic and natural? Let me see you find an external thrower who shall overtake it once more to throw it upward. (Galilei 1967, 236)

The thought experiment of the ball falling through a tunnel traversing the center of the earth has a long history. Its first known occurrence is in Plutarch's dialogue De facie (924a 8-12, b 4-8), a text with which Galileo was certainly acquainted (Casini 1984; Shea 2000; Fabbri 2012). Medieval and early modern Aristotelians often discussed the imaginary scenario in the context of their treatment of natural motion, proposing two different solutions. Some of them argued, in conformity with Aristotle's theory of gravity, that the ball would stop as soon as it reached the center of the earth, whereas others maintained, in accordance with the impetus theory, that the ball would oscillate around the center of the earth and would only come to rest once the impetus was exhausted (Hooykaas 1999; Palmerino 2011, 107-8).

Given that in the Dialogue Simplicio explicitly criticizes the impetus theory (Galilei 1967, 149-50), one would expect him to endorse the first solution, and yet he predicts that the cannon ball will "keep going a long way." It is at this point easy for Salviati to point out that Simplicio's solution is in contradiction with his proposed explanation of gravity and with the dichotomy between natural and violent motion, which would require the body to stop immediately at the center of the earth.

In this section, we have seen Salviati and Sagredo submit imaginary scenarios to Simplicio's scrutiny, with the intention of testing the cogency of Aristotle's theory of motion. Time and again the Aristotelian instinctively agrees with his interlocutors about what would happen and is subsequently led to realize that his proposed outcome is at odds with his theoretical as- 
sumptions. In the Dialogue, however, one also encounters text passages in which Simplicio appeals to thought experiments in order to confute Copernicus's heliocentric theory. Here, as we see in the following section, the interlocutors offer divergent answers to the "what would happen?" question.

3. Disagreeing on What Would Happen. The most famous example of a thought experiment that elicits different intuitions in Salviati and Simplicio is that of the ball dropped from the mast of a moving ship. In the second day of the Dialogue, Simplicio argues that, on a rotating earth, a body dropped from a high tower could not fall parallel to the tower because this would require having two natural motions at the same time, notably "a straight one towards the centre, and a circular one about the centre" (Galilei 1967, 14041). In support of this claim, he quotes "a very appropriate experiment," one that was adduced by the supporters of a geocentric cosmology as a proof of the impossibility of the daily rotation of the earth (Ariotti 1972; Grant 1984, 36-42). According to Simplicio, the behavior of a ball dropped from a tower on a moving earth should be inferred from that "of the stone dropped from the top of the mast of a ship, which falls to the foot of the mast when the ship is standing still, but falls as far from that same point when the ship is sailing" (Galilei 1967, 141). At this point, Galileo pens a very interesting dialogue, in which Salviati and Simplicio agree, first, that the "result of the earth" must be similar to "that on the ship" and, second, that if the stone dropped from the mast fell on the same place on the ship in motion as on the ship at rest, the experiment could be of no use in determining whether the ship is sailing.

SAlviati: Very good. Now, have you ever made this experiment of the ship?

SIMPLICIO: I have never made it, but I certainly believe that the authorities who adduced it had carefully observed it. . . .

SAlviati: You yourself are sufficient evidence that those authors may have offered it without having performed it, for you take it as certain without having done it. ... For anyone who does will find that the experiment shows exactly the opposite of what is written; that is, it will show that the stone always falls in the same place on the ship, whether the ship is standing still or moving with any speed you please. Therefore, the same cause holding good on the earth as on the ship, nothing can be inferred about the earth's motion or rest from the stone falling always perpendicularly to the foot of the tower. (Galilei 1967, 144-45)

It would seem as if Salviati is using the result of a real experiment to debunk an objection based on a pseudoexperiment. Surprisingly enough, he admits, however, that he has not performed the experiment either: 
SIMPLICIO: So you have not made a hundred tests, or even one? And yet you freely declare it to be certain? I shall retain my incredulity, and my own confidence that the experiment has been made by the most important authors who make use of it. . . .

SALVIATI: Without experiment, I am sure that the effect will happen as I tell you, because it must happen that way; and I might add that you yourself also know that it cannot happen otherwise, no matter how you may pretend not to know it. (Galilei 1967, 145)

It is likely that Galileo had in fact performed the experiment of the falling stone on a ship, as he himself acknowledged in his letter to Francesco Ingoli of 1624 (Galilei 1890-1909, 6:545). But why then did he feign to speak "without experiment"? He did so, I think, not out of prudence (Ariotti 1972, 2012) but rather in order to emphasize that theoretical assumptions influence our predictions concerning the behavior of physical bodies. Salviati tries to convince his Aristotelian interlocutor that horizontal motion and motion downward can be combined, as they are neither "destructive of one another, nor incompatible," but Simplicio retorts that the argument, although "very plausible in appearance, . . . is offset by a difficulty which is hard to overcome" because it was built on a premise "which will not lightly be granted by the Peripatetic school." It is in fact "contrary to Aristotle" to assume that a projectile "when separated from its origin retains the motion which was forcibly impressed upon it there" (Galilei 1967, 149).

This is not the only instance in the Dialogue in which Galileo shows that our contemplation of imaginary scenarios is theory laden. Something similar happens in a passage of the first day where Simplicio argues, in accordance with Aristotle's theory of natural places, that "if the terrestrial globe were forcibly removed from the place assigned to it by nature, it would return by a straight line." The Aristotelian infers this conclusion from the behavior of earthly bodies, which "removed from the whole ... return to their places naturally and spontaneously in a straight motion" (Galilei 1967, 33).

Salviati is quick to point out that Simplicio's conclusion is question begging, as it presupposes without proof "that the earth is de facto the centre of the universe." But what if gravity were a universal tendency of bodies to reunite with their whole? On the basis of this assumption, Salviati formulates a counter thought experiment:

SAlviati: Now just as all the parts of the earth mutually cooperate to form its whole ... why may we not believe that the sun, moon and other world bodies are also round in shape merely by a concordant instinct and natural tendencies of all their component parts? If at any time one of these parts were forcibly separated from the whole, is it not reasonable to believe that it would return spontaneously and by natural 
tendency? And in this manner we should conclude that straight motion is equally suitable to all world bodies. (Galilei 1967, 33-34)

This time, Simplicio does not offer an alternative interpretation of the imaginary scenario but simply refuses to make a prediction, arguing that what Salviati inquires into "would be the consequences of an impossibility." One could in fact never detach a part from the sun or the moon because, "as Aristotle also demonstrates, celestial bodies are invariant, impenetrable, and unbreakable." To this, the Aristotelian adds the puzzling remark that even if the situation described by Salviati should arise, "and the separated part did return to its whole, it would not return thus because of being heavy or light, since Aristotle also proves that celestial bodies are neither heavy nor light" (Galilei 1967, 34). Salviati cannot but castigate Simplicio once again for his "failure to perceive that Aristotle assumes what is in question. ... None of the conditions by which Aristotle distinguishes celestial from elemental bodies has any other foundation than that what he deduces from the difference in natural motion between the former and the latter" (35-37).

We have just seen a case in which Simplicio rejects a thought experiment put forward by Salviati on the grounds that the imagined situation could never take place. In another passage of the Dialogue, it is Galileo's spokesman who discards as nonsensical two thought experiments invoked by Simplicio to argue against the earth's natural motion.

In the second day of the Dialogue, Simplicio cites some passages from the Disquisitiones mathematicae (1614) of the German Jesuit Johann Georg Locher, which argue for what Finocchiaro has aptly called "the inexplicability of terrestrial rotation" $(2013,145)$. According to Locher, it was absurd to think that terrestrial objects could participate in the daily rotation of the earth and that the same internal principle could be responsible for the circular motion of birds, stones, arrows, snow, and fish, "their natures being most diverse" (Galilei 1967, 239-40). The Jesuit tried to strengthen his argument by invoking some counterfactual scenarios: What "if the earth should stop by the will of God?" Would flying birds and falling bodies continue to rotate? If not, then one could not regard their circular motion as natural; if yes, then "it would be truly remarkable if the seagull could not hover over the small fish, the skylark over her nest, and the crow over the snail and the rock" (Galilei 1967, 240). And what if the terrestrial globe were "reduced to nothing?" According to Locher, on the basis of Copernicus's theory, one should predict that "no hail or rain would fall from the clouds, but would only be carried naturally around; nor would any fire or flaming thing ascend, since in their view, probably, there is no fire above. ... Which, however, experience and reason refute" (Galilei 1967, 243).

Salviati refuses to answer Locher's questions, as he believes it makes little sense to discuss what would happen in situations that run counter to the 
ordinary course of nature (cf. Palmerino 2011, 118). In reaction to Locher's first argument Salviati observes that "if by God's will the earth should stop its diurnal whirling, the birds would do whatever that same will of God desired" (Galilei 1967, 240). As for the second argument, Salviati declares to know "just as much about what would happen after the earth was annihilated" as one could know "about what was going to take place on it and around it before it was created" (245). According to Salviati, experience cannot serve as a guide for the evaluation of scholastic arguments de potentia Dei absoluta, because "whatever begins with a Divine miracle or an angelic operation ... is not unlikely to do everything else by means of the same principle" (237). Galileo would agree with those philosophers of science who claim that in order to be effective, a thought experiment must describe a situation that can be handled on the basis of past experience (Kuhn 1977, 252). For nothing meaningful can be said about scenarios in which the laws of nature have been abrogated (Wilkes 1988, 15ff.; Rescher 2005, 152-53).

4. Conclusion. In an article bearing the evocative title "When Are Thought Experiments Poor Ones?" Jeanne Peijnenburg and David Atkinson have argued that thought experiments are successful "if they induce the same- - true or false - belief in the majority of people that are exposed to them" (2003, 307). They are, by contrast, "poor ones" if they lead to contradictory conclusions, if they beg the question, or if the scenarios imagined are too farfetched.

If one takes into account not only the epistemic power of imaginary scenarios but also their rhetorical and didactic value, the distinction between good and poor thought experiments becomes blurred. As we have seen in this article, only some of the thought experiments described in Galileo's dialogues induce the same belief in the three interlocutors. This type of thought experiments is usually invoked by Salviati or Sagredo to challenge some fundamental tenets of Aristotle's natural philosophy, such as the nature of gravity or the cause of projectile motion. Simplicio gives an instinctive, often not well-argued answer to the "what would happen?" question — an answer that the reader cannot but endorse. The Socratic dialogue that follows has the aim of showing that Simplicio's intuitive knowledge about the behavior of falling bodies and projectiles is in agreement with everyday experience but clashes with the principles of Aristotle's theory of motion.

In Galileo's dialogues one encounters, however, also "poor" thought experiments that elicit different intuitions in the three interlocutors. By letting his fictional characters $(a)$ draw diverging conclusions from an imaginary scenario, $(b)$ accuse one another of begging the question in their proposed solutions, and $(c)$ reject one another's thought experiments as meaningless or overly far-fetched, Galileo brings to light the incompatibility between the Aristotelian and Copernican worldviews. He suggests, moreover, that the eval- 
uation of an imaginary scenario is a theory-laden undertaking, as one's theoretical assumptions influence not only one's intuitions about what would happen but also one's ideas concerning the cogency and meaningfulness of a thought experiment.

\section{REFERENCES}

Ariotti, Pietro. 1972. "From the Top to the Foot of a Mast on a Moving Ship." Annals of Science 28:191-203.

Aristotle. 1929. Physics. Ed. and trans. Philip H. Wickstead and Francis M. Cornford. Cambridge, MA: Harvard University Press.

Atkinson, David, and Jeanne Peijnenburg. 2004. "Galileo and Prior Philosophy." Studies in History and Philosophy of Science A 35:115-36.

Brown, James R. 2000. "Thought Experiments." In A Companion to the Philosophy of Science, ed. William H. Newton-Smith, 528-31. Oxford: Blackwell.

Camilleri, Kristian. 2015. "Knowing What Would Happen: The Epistemic Strategies in Galileo's Thought Experiments." Studies in Historv and Philosophy of Science A 54:102-12.

Cantor, Geoffrey. 1989. "The Rhetoric of Experiment." In The Uses of Experiment: Studies in the Natural Sciences, ed. David Gooding and Trevor Pinch, 159-80. Cambridge: Cambridge University Press.

Casini, Paolo. 1984. "Il Dialogo di Galileo e la luna di Plutarco." In Novità celesti e crisi del sapere: Atti del convegno internazionale di studi galileiani, ed. Paolo Galluzzi, 57-62. Florence: Giunti Barbera.

Fabbri, Natacha. 2012. "The Moon as Another Earth: What Galileo Owes to Plutarch." Galilaeana 9:103-35.

Finocchiaro, Maurice. 2013. The Routledge Guidebook to Galileo's Dialogue. London: Routledge. Galilei, Galileo. 1890-1909. Le Opere. 20 vols., ed. Antonio Favaro. Florence: Barbera. 1967. Dialogue Concerning the Two Chief World Systems. 2nd ed., ed. and trans. Stillman Drake. Berkeley: University of California Press. - 1989. Two New Sciences. Ed. and trans. Stillman Drake. Toronto: Wall \& Emerson.

Gendler, Tamar. 1998. "Galileo and the Indispensability of Scientific Thought Experiment." British Journal for the Philosophy of Science 49:397-424.

Grant, Edward. 1984. "In Defense of the Earth's Centrality and Immobility: Scholastic Reaction to Copernicanism in the Seventeenth Century." Transactions of the American Philosophical Society 74:1-69.

- 2002. "Medieval Natural Philosophy: Empiricism without Observation.” In The Dynamics of Aristotelian Natural Philosophy from Antiquity to the Seventeenth Century, ed. Cees Leijenhorst, Christoph Lüthy, and Hans Thijssen, 141-68. Leiden: Brill.

Hooykaas, Reijer. 1999. "A Tunnel through the Earth.” In Fact, Faith and Fiction in the Development of Science: The Gifford Lectures Given in the University of St. Andrews, 1976, 117-45. Dordrecht: Kluwer.

Jardine, Nicholas. 1991. "Demonstration, Dialectic, and Rhetoric in Galileo's Dialogue." In The Shapes of Knowledge from the Renaissance to the Enlightenment, ed. Donald R. Kelley and Richard H. Popkin, 101-21. Dordrecht: Kluwer.

Kuhn, Thomas. 1977. The Essential Tension: Selected Studies in Scientific Tradition and Change. Chicago: University of Chicago Press.

Murdoch, John E. 1982. "The Analytic Character of Late Medieval Learning: Natural Philosophy without Nature." In Approaches to Nature in the Middle Ages, ed. Lawrence Roberts, 171213. Binghamton, NY: Center for Medieval and Renaissance Texts and Studies.

Norton, John. 1996. “Are Thought Experiments Just What You Thought?” Canadian Journal of Philosophv 26:333-66.

. 2004. "Why Thought Experiments Do Not Transcend Empiricism." In Contemporary Debates in the Philosophy of Science, ed. Christopher Hitchcock, 44-66. Oxford: Blackwell. 
Palmerino, Carla Rita. 2011. "Galileo's Use of Medieval Thought Experiments.” In Thought Experiments in Methodological and Historical Contexts, ed. Katarina Ierodiakonou and Sophie Roux, 101-26. Leiden: Brill.

Palmieri, Paolo. 2005. "'Spuntar lo scoglio più duro': Did Galileo Ever Think the Most Beautiful Thought Experiment in the History of Science?" Studies in Historv and Philosophy of Science A 36:223-40.

Peijnenburg, Jeanne, and David Atkinson. 2003. "When Are Thought Experiments Poor Ones?" Journal for General Philosophy of Science 34:305-22.

Rescher, Nicholas. 2005. What If? Thought Experimentation in Philosophy. New Brunswick, NJ: Transaction.

Shea, William. 2000. "Looking at the Moon as Another Earth: Terrestrial Analogies and SeventeenthCentury Telescopes." In Metaphor and Analogy in the Sciences, ed. Fernand Hallyn, 83-104. Dordrecht: Kluwer.

Wilkes, Kathleen. 1988. Real People: Personal Identity without Thought Experiments. Oxford: Oxford University Press. 